\title{
A Distance Geometry Approach to the Singularity Analysis of 3R Robots
}

\author{
Federico Thomas \\ Institut de Robòtica i Informàtica Industrial (CSIC-UPC) \\ Llorens Artigas 4-6, 08028 Barcelona, Spain \\ fthomas@iri.upc.edu
}

\begin{abstract}
This paper shows how the computation of the singularity locus of a $3 R$ robot can be reduced to the analysis of the relative position of two coplanar ellipses. Since the relative position of two conics is a projective invariant, and the basic projective geometric invariants are determinants, it is not surprising that, using Distance Geometry, the computation of the singularity locus of a $3 R$ robot can be fully expressed in terms of determinants. Geometric invariants have the benefit of simplifying symbolic manipulations. This paper shows how their use leads to a simpler characterization, compared to previous approaches, of the cusps and nodes in the singularity loci of $3 R$ robots.
\end{abstract}

Keywords: Wrist-partitioned robots, 3R robots, cuspidal robots, singularities, cusps, nodes, Distance Geometry, coordinatefree formulations.

\section{Introduction}

Wrist-partitioned robots can be thought of as having two parts. The first three links of the robot, or regional part, provide the translation of the robot's hand, and the last three are responsible for providing the hand's orientation. The regional part of most wrist-partitioned robots used in Industry is based on a 3R robot having important simplifying geometric conditions to make its inverse kinematics reduce to the solution of quadratic equations. One important consequence of these simplifications is that these robots have to pass through a singularity to change their working mode (change from one inverse kinematic solution to another). Nevertheless, this behavior is not general at all [1]. It has been shown how a generic $3 \mathrm{R}$ robot can change its working mode, without meeting any singularity, if at least one point in its workspace has exactly three inverse kinematic solutions (corresponding to a cusp point in its singularity locus). This observation reveals that a precise understanding of the nature of the singularity curves of generic 3R robots can assist in the design of industrial robots [2]. This is the motivation behind the work presented in this paper where it is shown how, using Distance Geometry, a simple characterization of the singularity loci of $3 \mathrm{R}$ robots is possible, thus departing from the mainstream dominated by the use of algebraic geometry methods applied on trivial algebraizations of the problem.

The forward kinematics of a 3R robot can be solved in terms of its Denavit and Hartenberg parameters and $4 \times 4$ homogeneous matrices [3]. This leads to a system of equations of the form:

$$
\left.\begin{array}{l}
x=f_{1}\left(\theta_{1}, \theta_{2}, \theta_{3}\right) \\
y=f_{2}\left(\theta_{1}, \theta_{2}, \theta_{3}\right) \\
z=f_{3}\left(\theta_{1}, \theta_{2}, \theta_{3}\right)
\end{array}\right\}
$$

where $(x, y, z)$ are the Cartesian coordinates of the robot's end-effector. By properly locating the world's reference frame and giving the end-effector center in cylindrical coordinates $(\phi, \rho, z)$, a symmetry with respect to the first joint axis can be exploited to simplify (1) (see Fig. 1) [9]. Using this representation, in 1968, Pieper proposed a technique to eliminate $\theta_{1}$ and $\theta_{2}$ from the above three equations to obtain an expression of the form [4]:

$$
F\left(\rho, z, \theta_{3}\right)=0 .
$$




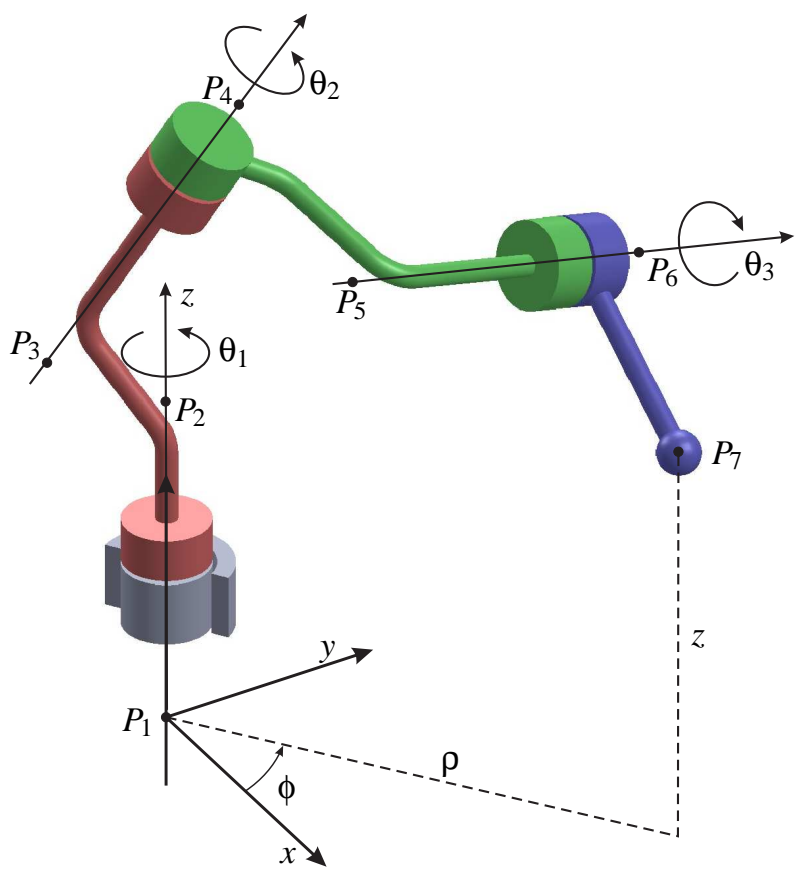

Fig. 1. A 3R robot and associated notation.

This formula has been the basis of most subsequent studies on the kinematics of $3 \mathrm{R}$ robots. Its analysis shows that:

- For fixed values of $\theta_{3}$, it represents an ellipse in the plane defined by $\rho$ and $z$.

- For fixed values of $\rho$ and $z$, it leads to a quartic polynomial in $t=\arctan \left(\theta_{3} / 2\right)$.

Each of the four roots of the quartic polynomial in $t$ determines a set of joint angles $\left(\theta_{1}, \theta_{2}, \theta_{3}\right)$. This solves the inverse kinematics of $3 \mathrm{R}$ robots in closed-form and permits to conclude that they can have up to four working modes. Nevertheless, the tangent half-angle substitution introduces some inconveniences [5]. As an alternative, in 1993, Smith and Lipkin reformulated (2) as:

$$
\left.\begin{array}{rl}
G(\rho, z, s, c) & =0 \\
s^{2}+c^{2} & =1
\end{array}\right\}
$$

where $s=\sin \theta_{3}$ and $c=\cos \theta_{3}[6,7]$. For fixed values of $\rho$ and $z$ the solutions to the above system of equations can be seen as the intersections of an ellipse and a circle. Having represented the inverse kinematics as intersecting conics, the properties of conics can then be used to derive the properties of the manipulator's workspace. The approach presented in this paper keeps some parallelism with this one because, as we will see, it also relies on the properties of conics.

As we already mentioned, the characterization of the singularity loci of $3 \mathrm{R}$ robots is a fundamental issue. To obtain them using (2) we can either:

- obtain the envelope of the family of ellipses generated as $\theta_{3}$ is swept in the range $[0, \infty)[8]$; or

- impose two equal roots to $F\left(\rho, z, \theta_{3}\right)=0$ as an expression in $\theta_{3}$.

Although the geometric interpretation is different, both methods are algebraically equivalent. Both lead to the following system of equations:

$$
\left.\begin{array}{l}
F\left(\rho, z, \theta_{3}\right)=0 \\
F_{\theta_{3}}\left(\rho, z, \theta_{3}\right)=0
\end{array}\right\}
$$

where $F_{\theta_{3}}$ denotes the partial derivative of $F$ with respect to $\theta_{3}$. By eliminating $\theta_{3}$ in the above system, it is possible to obtain the resultant

$$
H(\rho, z)=0,
$$



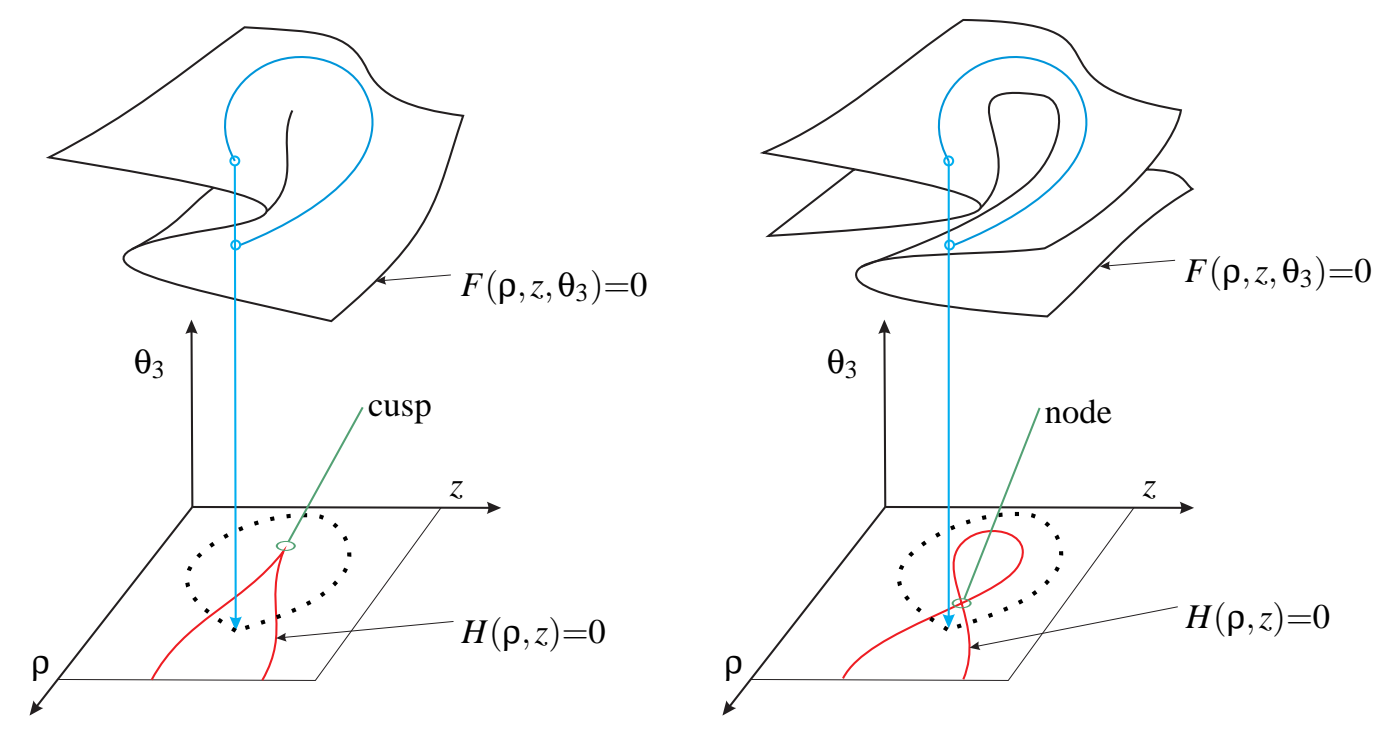

Fig. 2. Encircling a cusp (left) or an $\alpha$-curve (right) allows, under some circumstances, to change the working mode of a robot without trespassing a singularity. The curves in red represent part of the singularity loci.

which can be seen as the equation of a curve, in implicit form, resulting from projecting orthographically, onto the plane defined by $\rho$ and $z$, those points of $F\left(\rho, z, \theta_{3}\right)=0$ where the direction defined by $\theta_{3}$ is tangent to the surface (see Fig. 2). These singularity curves, for 3R robots with orthogonal axes, are known to exhibit up to 8 cusps and up to 4 nodes $[1,10]$. They divide the plane defined by $\rho$ and $z$ into regions where the number of working modes is constant and, depending on how these regions are distributed, interesting results can be drawn. For example, it has been shown that it is possible to change from one working mode to another one without meeting any singularity at least in two situations: by encircling a cusp [1] or by encircling a so-called $\alpha$-curve [11] (see Fig. 2). Thus, it is important to locate the nodes and cusps in these singularity curves.

Equation (5) is a description of the singularity locus in implicit form. In 1989, Ceccarelli [8] showed how to obtain this curve in parametric form by first eliminating $\rho$, thus transforming the system in (4) into

$$
\left.\begin{array}{l}
z=g_{1}\left(\theta_{3}\right) \\
\rho=g_{2}\left(z, \theta_{3}\right)
\end{array}\right\}
$$

Based on this formulation, Saramago et al. [12] proposed a general analytical condition to deduce the existence of cusps and nodes in the singularity locus. Nevertheless, this formulation has received little attention and the computation of cusps has been dominated by the technique consisting in imposing three equal roots to (2), that is, in solving the following system of equations:

$$
\left.\begin{array}{l}
F(\rho, z, t)=0 \\
F_{t}(\rho, z, t)=0 \\
F_{t t}(\rho, z, t)=0
\end{array}\right\}
$$

where $F_{t}$ and $F_{t t}$ denote the first and the second partial derivative of $F$ with respect to $t$, respectively (where $t=\arctan \left(\theta_{3} / 2\right.$ ), as above) $[10,13,14]$. The derivation of a general condition for the existence of cusps was also attempted in [13] using the determinant of the Jacobian matrix. When deriving the singularity locus from this determinant, the result is represented as a curve in the plane defined by $\theta_{2}$ and $\theta_{3}$ - that is, the configuration space of the robot—(see [15] and the references therein). Then, this curve can be mapped onto the plane defined by $\rho$ and $z$ using the forward kinematics of the manipulator. In both cases, the analysis leads, in the general case, to unwieldy expressions that drive most authors to limit their analyses to $3 \mathrm{R}$ robots with orthogonal axes. The results presented here concern the computation of nodes and cusps in the general case, thus extending some preliminary results presented in [16] which were limited to the computation of cusps.

The rest of the paper is organized as follows. Section 2 describes how, using Distance Geometry, the inverse kinematics of a $3 \mathrm{R}$ robot boils down to compute the intersection of two ellipses. Section 3 shows how the singularity locus of a 3R robot can be computed from the pencil of conics defined by these two ellipses. Then, based on the fact that cusps and nodes in this locus correspond to osculating contacts and double contacts between both ellipses, respectively, Sections 4 and 5 

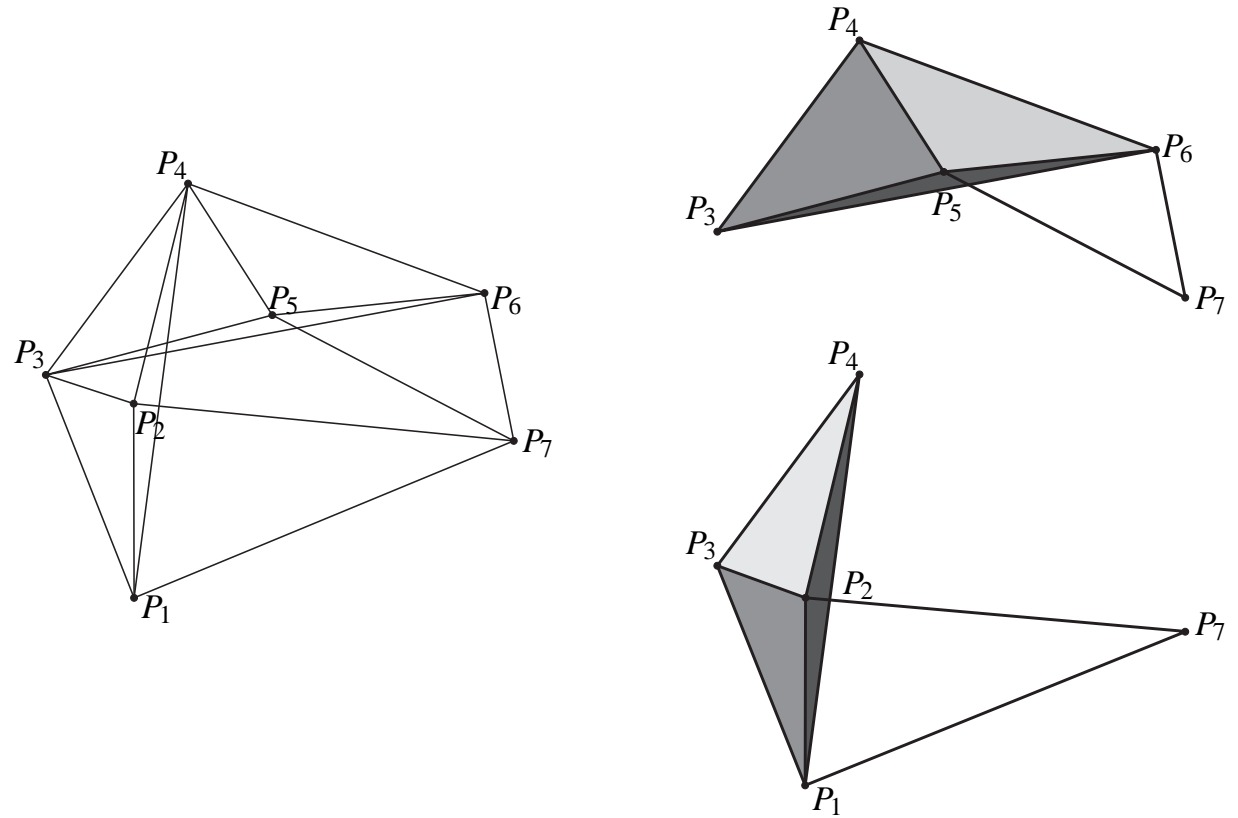

Fig. 3. Left: The equivalent bar-and-joint framework associated with the $3 R$ robot in Fig. 1. Right: This framework can be split by the plane defined by $P_{3}, P_{4}$ and $P_{7}$ into two subassemblies, each containing a tetrahedron (shown as a shaded volume) and a triangle.

derive algebraic conditions for their computation. All these ideas are illustrated through an example in Section 6. Section 7 summarizes the main points and gives prospective points for further research.

\section{Distance-based formulation}

When fixing the location of a 3R robot end-effector, the first and last links can be seen as articulated through a spherical joint centered at the robot's end-effector and the inverse kinematics problem is equivalent to that of finding the valid configurations of a closed loop of four pairwise articulated links through three revolute joints and one spherical joint.

A link connecting two revolute axes can be modeled by taking two points on each of these axes, and by connecting them all with rigid bars to form a tetrahedron. A link connecting a revolute axis and a spherical joint can be modeled by taking two points on the revolute joint axis and the center of the spherical joint, and by connecting them all with rigid bars to form a triangle. In this way, the $3 \mathrm{R}$ robot shown in Fig. 1 can be modeled as the bar-and-joint framework shown in Fig. 3(left). In this conversion we are loosing an important information: the orientation of the two tetrahedra. Nevertheless, given a valid configuration of the obtained bar-and-joint framework, we can derive another valid configuration with the desired orientations for the two tetrahedra. Indeed, observe that $P_{3}, P_{4}$, and $P_{7}$ defines a plane that divides the framework in two halves and the mirror projection of any of these two halves with respect to this plane changes the orientation of the corresponding tetrahedron while preserving all bar lengths. As a consequence, any valid configuration for the framework translates into a valid configuration for the $3 \mathrm{R}$ robot.

The distances between the set of points $\left\{P_{1}, P_{2}, P_{3}, P_{4}, P_{7}\right\}$ or $\left\{P_{3}, P_{4}, P_{5}, P_{6}, P_{7}\right\}$ are not independent because they are embedded in $\mathbb{R}^{3}$. This dependency, using the theory of Cayley-Menger determinants [17], translates into the following algebraic conditions:

$$
\left|\begin{array}{cccccc}
0 & 1 & 1 & 1 & 1 & 1 \\
1 & 0 & s_{1,2} & s_{1,3} & s_{1,4} & s_{1,7} \\
1 & s_{2,1} & 0 & s_{2,3} & s_{2,4} & s_{2,7} \\
1 & s_{3,1} & s_{3,2} & 0 & s_{3,4} & s_{3,7} \\
1 & s_{4,1} & s_{4,2} & s_{4,3} & 0 & s_{4,7} \\
1 & s_{7,1} & s_{7,2} & s_{7,3} & s_{7,4} & 0
\end{array}\right|=0 \quad \text { and }\left|\begin{array}{cccccc}
0 & 1 & 1 & 1 & 1 & 1 \\
1 & 0 & s_{3,4} & s_{3,5} & s_{3,6} & s_{3,7} \\
1 & s_{4,3} & 0 & s_{4,5} & s_{4,6} & s_{4,7} \\
1 & s_{5,3} & s_{5,4} & 0 & s_{5,6} & s_{5,7} \\
1 & s_{6,3} & s_{6,4} & s_{6,5} & 0 & s_{6,7} \\
1 & s_{7,3} & s_{7,4} & s_{7,5} & s_{7,6} & 0
\end{array}\right|=0
$$

where $s_{i, j}$ stands for the squared distance between $P_{i}$ and $P_{j}$. The above two equations are quadratic forms in the unknown distances $s_{3,7}$ and $s_{4,7}$. They actually represent two conic sections, $\mathcal{A}: \mathbf{x A \mathbf { A }} \mathbf{x}^{T}=0$ and $\mathcal{B}: \mathbf{x B \mathbf { x }} T=0$, where $\mathbf{x}=\left(s_{3,7}, s_{4,7}, 1\right)$ 
and

$$
\mathbf{A}=\left(\begin{array}{lll}
a_{1} & c_{1} & d_{1} \\
c_{1} & b_{1} & e_{1} \\
d_{1} & e_{1} & f_{1}
\end{array}\right) \quad \text { and } \quad \mathbf{B}=\left(\begin{array}{lll}
a_{2} & c_{2} & d_{2} \\
c_{2} & b_{2} & e_{2} \\
d_{2} & e_{2} & f_{2}
\end{array}\right)
$$

The entries of $\mathbf{A}$ and $\mathbf{B}$ can, in turn, be expressed in terms of determinants as follows:

$$
\begin{aligned}
& a_{1}=-\left|\begin{array}{cccc}
0 & 1 & 1 & 1 \\
1 & 0 & s_{1,2} & s_{1,4} \\
1 & s_{1,2} & 0 & s_{2,4} \\
1 & s_{1,4} & s_{2,4} & 0
\end{array}\right|, \quad b_{1}=-\left|\begin{array}{cccc}
0 & 1 & 1 & 1 \\
1 & 0 & s_{1,2} & s_{1,3} \\
1 & s_{1,2} & 0 & s_{2,3} \\
1 & s_{1,3} & s_{2,3} & 0
\end{array}\right|, \quad c_{1}=\left|\begin{array}{cccc}
0 & 1 & 1 & 1 \\
1 & 0 & s_{1,2} & s_{1,3} \\
1 & s_{1,2} & 0 & s_{2,3} \\
1 & s_{1,4} & s_{2,4} & s_{3,4}
\end{array}\right|, \\
& d_{1}=-\left|\begin{array}{ccccc}
0 & 1 & 1 & 1 & 1 \\
1 & 0 & s_{1,2} & s_{1,4} & s_{1,7} \\
1 & s_{1,2} & 0 & s_{2,4} & s_{2,7} \\
1 & s_{1,4} & s_{2,4} & 0 & 0 \\
1 & s_{1,3} & s_{2,3} & s_{3,4} & 0
\end{array}\right|, \quad e_{1}=-\left|\begin{array}{ccccc}
0 & 1 & 1 & 1 & 1 \\
1 & 0 & s_{1,2} & s_{1,3} & s_{1,7} \\
1 & s_{1,2} & 0 & s_{2,3} & s_{2,7} \\
1 & s_{1,3} & s_{2,3} & 0 & 0 \\
1 & s_{1,4} & s_{2,4} & s_{3,4} & 0
\end{array}\right|, \quad f_{1}=\left|\begin{array}{cccccc}
0 & 1 & 1 & 1 & 1 & 1 \\
1 & 0 & s_{1,2} & s_{1,3} & s_{1,4} & s_{1,7} \\
\hline 1 & s_{1,2} & 0 & s_{2,3} & s_{2,4} & s_{2,7} \\
1 & s_{1,3} & s_{2,3} & 0 & s_{2,4} & 0 \\
1 & s_{1,4} & s_{2,4} & s_{3,4} & 0 & 0 \\
1 & s_{1,7} & s_{2,7} & 0 & 0 & 0
\end{array}\right|, \\
& a_{2}=-\left|\begin{array}{cccc}
0 & 1 & 1 & 1 \\
1 & 0 & s_{5,6} & s_{5,4} \\
1 & s_{5,6} & 0 & s_{6,4} \\
1 & s_{5,4} & s_{6,4} & 0
\end{array}\right|, \quad b_{2}=-\left|\begin{array}{cccc}
0 & 1 & 1 & 1 \\
1 & 0 & s_{5,6} & s_{5,3} \\
1 & s_{5,6} & 0 & s_{6,3} \\
1 & s_{5,3} & s_{6,3} & 0
\end{array}\right|, \quad c_{2}=\left|\begin{array}{cccc}
0 & 1 & 1 & 1 \\
1 & 0 & s_{5,6} & s_{5,3} \\
1 & s_{5,6} & 0 & s_{6,3} \\
1 & s_{5,4} & s_{6,4} & s_{3,4}
\end{array}\right|, \\
& d_{2}=-\left|\begin{array}{ccccc}
0 & 1 & 1 & 1 & 1 \\
1 & 0 & s_{5,6} & s_{5,4} & s_{5,7} \\
1 & s_{5,6} & 0 & s_{2,4} & s_{6,7} \\
1 & s_{5,4} & s_{6,4} & 0 & 0 \\
1 & s_{5,3} & s_{6,3} & s_{3,4} & 0
\end{array}\right|, \quad e_{2}=-\left|\begin{array}{ccccc}
0 & 1 & 1 & 1 & 1 \\
1 & 0 & s_{5,6} & s_{5,3} & s_{5,7} \\
1 & s_{5,6} & 0 & s_{6,3} & s_{6,7} \\
1 & s_{5,3} & s_{6,3} & 0 & 0 \\
1 & s_{5,4} & s_{6,4} & s_{3,4} & 0
\end{array}\right|, \quad f_{2}=\left|\begin{array}{cccccc}
0 & 1 & 1 & 1 & 1 & 1 \\
1 & 0 & s_{5,6} & s_{5,3} & s_{5,4} & s_{5,7} \\
1 & s_{5,6} & 0 & s_{6,3} & s_{6,4} & s_{6,7} \\
1 & s_{5,3} & s_{6,3} & 0 & s_{3,4} & 0 \\
1 & s_{5,4} & s_{6,4} & s_{3,4} & 0 & 0 \\
1 & s_{5,7} & s_{6,7} & 0 & 0 & 0
\end{array}\right| .
\end{aligned}
$$

All entries in the above determinants are constant for a given manipulator, except $s_{1,7}$ and $s_{2,7}$ that depend on the location of the robot's end-effector. Therefore, all entries of $\mathbf{A}$ and $\mathbf{B}$ are constant, except $d_{1}$ and $e_{1}$ that depend linearly on $s_{1,7}$ and $s_{2,7}$, and $f_{1}$ that depends on them quadratically.

We can now compute the intersection of $\mathcal{A}$ and $\mathcal{B}$ to obtain sets of distances $\left(s_{1,7}, s_{2,7}\right)$ compatible with all other distances. It is important to note that, given the orientation of the tetrahedra with vertex sets $\left\{P_{1}, P_{2}, P_{3}, P_{4}\right\}$ and $\left\{P_{3}, P_{4}, P_{5}, P_{6}\right\}$, every solution for $\left(s_{1,7}, s_{2,7}\right)$ leads to a unique solution for $\left(\theta_{2}, \theta_{3}\right)$. Thus, solving the inverse kinematics of a $3 \mathrm{R}$ robot reduces to calculate the intersections of two conic sections. Since two conic sections intersect in up to four real points, this indicates that it is necessary to solve a quartic polynomial, as it was already well-known. Actually, the geometric interpretation of the solution of a quartic polynomial is usually presented as the intersection of two conics [18].

Some of the above determinants have a direct geometric interpretation using the properties of Cayley-Menger determinants [17]. Using these properties, the affine invariants of the conics $\mathcal{A}$ and $\mathcal{B}$ (see [19, pp. 176-188]) can be expressed as:

$$
\begin{aligned}
& T_{\mathbf{A}}=a_{1}+b_{1}=16\left(A_{1,2,4}^{2}+A_{1,2,3}^{2}\right), \\
& E_{\mathbf{A}}=\left|\begin{array}{ll}
a_{1} & c_{1} \\
c_{1} & b_{1}
\end{array}\right|=\left(16 A_{1,2,4} A_{1,2,3}\right)^{2}\left(1-\cos \phi_{1,2,3,4}\right), \\
& D_{\mathbf{A}}=\left|\begin{array}{lll}
a_{1} & c_{1} & d_{1} \\
c_{1} & b_{1} & e_{1} \\
d_{1} & e_{1} & f_{1}
\end{array}\right|=\left|\begin{array}{ccccc}
0 & 1 & 1 & 1 & 1 \\
1 & 0 & s_{1,2} & s_{1,3} & s_{1,4} \\
1 & s_{1,2} & 0 & s_{2,3} & s_{2,4} \\
1 & s_{1,3} & s_{2,3} & 0 & s_{3,4} \\
1 & s_{1,4} & s_{2,4} & s_{3,4} & 0
\end{array}\right| \begin{array}{cccc}
0 & 1 & 1 & 1 \\
1 & 0 & s_{1,2} & s_{1,7} \\
1 & s_{1,2} & 0 & s_{2,7} \\
1 & s_{1,7} & s_{2,7} & 0
\end{array} \mid=\left(288 V_{1,2,3,4}^{2}\right)^{2}\left(-16 A_{1,2,7}^{2}\right)=-4608^{2} V_{1,2,3,4}^{4} A_{1,2,7}^{2}
\end{aligned}
$$


and

$$
\begin{aligned}
& T_{\mathbf{B}}=a_{2}+b_{2}=16\left(A_{4,5,6}^{2}+A_{3,5,6}^{2}\right), \\
& E_{\mathbf{B}}=\left|\begin{array}{ll}
a_{2} & c_{2} \\
c_{2} & b_{2}
\end{array}\right|=\left(16 A_{5,6,4} A_{5,6,3}\right)^{2}\left(1-\cos \phi_{5,6,3,4}\right), \\
& D_{\mathbf{B}}=\left|\begin{array}{lll}
a_{2} & c_{2} & d_{2} \\
c_{2} & b_{2} & e_{2} \\
d_{2} & e_{2} & f_{2}
\end{array}\right|=\left|\begin{array}{ccccc}
0 & 1 & 1 & 1 & 1 \\
1 & 0 & s_{3,4} & s_{3,5} & s_{3,6} \\
1 & s_{3,4} & 0 & s_{4,5} & s_{4,6} \\
1 & s_{3,5} & s_{4,5} & 0 & s_{5,6} \\
1 & s_{3,6} & s_{4,6} & s_{5,6} & 0
\end{array}\right|\left|\begin{array}{cccc}
0 & 1 & 1 & 1 \\
1 & 0 & s_{5,6} & s_{5,7} \\
1 & s_{5,6} & 0 & s_{6,7} \\
1 & s_{5,7} & s_{6,7} & 0
\end{array}\right|=\left(288 V_{3,4,5,6}^{2}\right)^{2}\left(-16 A_{5,6,7}^{2}\right)=-4608^{2} V_{3,4,5,6}^{4} A_{5,6,7}^{2},
\end{aligned}
$$

respectively, where $V_{i, j, k, l}$ stands for the volume of the tetrahedron with vertex set $\left\{P_{i}, P_{j}, P_{k}, P_{l}\right\}, A_{i, j, l}$ for the area of $\triangle P_{i} P_{j} P_{l}$, and $\phi_{i, j, l, k} \in[0, \pi]$ for the dihedral angle between $\triangle P_{i} P_{j} P_{l}$ and $\triangle P_{i} P_{j} P_{k}$ hinged along $\frac{P_{i}, P_{j}}{\text {. }}$

Now, observe that:

1. The first and the second rotation axes coincide if, and only if, $A_{1,2,3}=0$ and $A_{1,2,4}=0$.

2. The second and the third rotation axes coincide if, and only if, $A_{4,5,6}=0$ and $A_{3,5,6}=0$.

3. The robot's end-effector center lies on the third rotation axis if, and only if, $A_{5,6,7}=0$.

4. The first and the second rotation axes intersect or are parallel if, and only if, $V_{1,2,3,4}=0$.

5. The second and the third rotation axes intersect or are parallel if, and only if, $V_{3,4,5,6}=0$.

If we exclude from our analysis the above five degenerate cases in which the analysis could be performed using less than seven points, then $T_{\mathbf{B}}>0, E_{\mathbf{B}}>0, D_{\mathbf{B}}<0, T_{\mathbf{A}}>0, E_{\mathbf{A}}>0$, and

$$
\operatorname{sign}\left(D_{\mathbf{A}}\right)=\operatorname{sign}[\operatorname{det}(\mathbf{A})]=\operatorname{sign}\left|\begin{array}{cccc}
0 & 1 & 1 & 1 \\
1 & 0 & s_{1,2} & s_{1,7} \\
1 & s_{1,2} & 0 & s_{2,7} \\
1 & s_{1,7} & s_{2,7} & 0
\end{array}\right|
$$

As a consequence, $\mathcal{B}$ is a real ellipse that depends only on the robot's geometry, and $\mathcal{A}$ is a ellipse that changes with the location of the robot's end-effector as follows:

- it is real if $\operatorname{det}(\mathbf{A})<0$ (in this case, the input squared distances $s_{1,7}$ and $s_{2,7}$ allow $P_{1}, P_{2}$ and $P_{7}$ to form a non-degenerate triangle);

- it degenerates into a point if $\operatorname{det}(\mathbf{A})=0$ (in this case, $P_{1}, P_{2}$ and $P_{7}$ are aligned or, in other words, the center of the robot's end-effector lies on the first rotation axis); and

- it is imaginary if $\operatorname{det}(\mathbf{A})>0$ (in this case, the input squared distances $s_{1,7}$ and $s_{2,7}$ do not allow $P_{1}, P_{2}$ and $P_{7}$ to form a triangle).

In what follows, we will obviously limit our analysis to the region of the plane defined by $\left(s_{1,7}, s_{2,7}\right)$ where $\operatorname{det}(\mathbf{A}) \leq 0$.

\section{Singularities}

A singularity occurs when we have a repeated solution of the inverse kinematics, that is, when $\mathcal{A}$ and $\mathcal{B}$ are tangent. The positional relationship between $\mathcal{A}$ and $\mathcal{B}$ can be derived from the study of the pencil of conics they define, that is, from the family of conics defined by $\mathbf{p}^{T}(\lambda \mathbf{A}+\mathbf{B}) \mathbf{p}=0, \lambda \in\{\mathbb{R} \cup \infty\}$ (see [20] for an introductory explanation). The values of $\lambda$ for which a conic of this pencil is degenerate correspond to those in which

$$
f(\lambda)=\operatorname{det}(\lambda \mathbf{A}+\mathbf{B})=l_{3} \lambda^{3}+3 l_{2} \lambda^{2}+3 l_{1} \lambda+l_{0}=0,
$$


where the coefficients $l_{i}, i=0,1,2,3$, can be expressed in a neat and elegant way as [21, p. 274] [22, p. 191]:

$$
\begin{aligned}
l_{3} & =\left|\begin{array}{lll}
a_{1} & c_{1} & d_{1} \\
c_{1} & b_{1} & e_{1} \\
d_{1} & e_{1} & f_{1}
\end{array}\right|=\operatorname{det}(\mathbf{A}), \\
3 l_{2} & =\left|\begin{array}{lll}
a_{2} & c_{1} & d_{1} \\
c_{2} & b_{1} & e_{1} \\
d_{2} & e_{1} & f_{1}
\end{array}\right|+\left|\begin{array}{lll}
a_{1} & c_{2} & d_{1} \\
c_{1} & b_{2} & e_{1} \\
d_{1} & e_{2} & f_{1}
\end{array}\right|+\left|\begin{array}{lll}
a_{1} & c_{1} & d_{2} \\
c_{1} & b_{1} & e_{2} \\
d_{1} & e_{1} & f_{2}
\end{array}\right|, \\
3 l_{1} & =\left|\begin{array}{lll}
a_{1} & c_{2} & d_{2} \\
c_{1} & b_{2} & e_{2} \\
d_{1} & e_{2} & f_{2}
\end{array}\right|+\left|\begin{array}{lll}
a_{2} & c_{1} & d_{2} \\
c_{2} & b_{1} & e_{2} \\
d_{2} & e_{1} & f_{2}
\end{array}\right|+\left|\begin{array}{lll}
a_{2} & c_{2} & d_{1} \\
c_{2} & b_{2} & e_{1} \\
d_{2} & e_{2} & f_{1}
\end{array}\right|, \\
l_{0} & =\left|\begin{array}{lll}
a_{2} & c_{2} & d_{2} \\
c_{2} & b_{2} & e_{2} \\
d_{2} & e_{2} & f_{2}
\end{array}\right|=\operatorname{det}(\mathbf{B}) .
\end{aligned}
$$

The above polynomial in $\lambda$ is known as the generalized characteristic polynomial of the pencil.

When the two ellipses defining the pencil are tangent, two of the three degenerate conics in the pencil become identical. This implies that the characteristic equation of the pencil has a repeated root for $\lambda[23]$. By definition, $f(\lambda)=0$ has a multiple root if, and only if, its discriminant, say $\Delta$, vanishes. Furthermore, it can be shown that $f(\lambda)=0$ has three simple real roots if $\Delta>0$, and $f(\lambda)=0$ has two complex conjugate roots and a real root if $\Delta<0$. Then, the roots of $\Delta=0$ give information on the positional relationship between $\mathcal{A}$ and $\mathcal{B}$. Actually, the sign of the discriminant $\Delta$ gives information about the order of accessibility of the manipulator's workspace. It permits to decompose it in the following three regions:

- $\Delta<0$ corresponds to a two-way accessible region;

- $\Delta=0$ corresponds to the singularities of the manipulator; and

- $\Delta>0$ corresponds to a four-way accessible region or an inaccessible region.

To be rigorous, we should say that $\Delta=0$ corresponds to the singularities of the robot ( $\mathcal{A}$ and $\mathcal{B}$ are tangent) and to isolated points, unreachable by the robot, where $\mathcal{A}$ and $\mathcal{B}$ have a double contact in the complex domain (see [24] for an explanation of this fact). We will ignore these points for the moment, but they will reappear when computing the nodes of the singularity locus.

The standard expression of the discriminant of a cubic gives little insight into the structure of our problem [25]. Fortunately, a much more convenient expression, that will reveal very important for the computation of cusps, can be found in [26]. It reads as follows:

$$
\Delta=\left|\begin{array}{cc}
2 \delta_{1} & \delta_{2} \\
\delta_{2} & 2 \delta_{3}
\end{array}\right|
$$

where

$$
\delta_{1}=\left|\begin{array}{ll}
l_{3} & l_{2} \\
l_{2} & l_{1}
\end{array}\right|, \quad \delta_{2}=\left|\begin{array}{ll}
l_{3} & l_{1} \\
l_{2} & l_{0}
\end{array}\right|, \quad \text { and } \quad \delta_{3}=\left|\begin{array}{ll}
l_{2} & l_{1} \\
l_{1} & l_{0}
\end{array}\right|
$$

Observe that the condition of singularity $\Delta=0$ is expressed as a determinant of determinants of determinants of determinants (four levels of nested determinants). It can be shown that the elements of the third level of determinants depend quadratically on $s_{3,7}$ and $s_{4,7}$. Then, since the two outer levels of determinants are quadratic with respect to their elements, the singularity locus can be displayed as a curve of order $2^{3}$ in the plane defined by $s_{1,7}$ and $s_{2,7}$.

The singularity locus correspond to those points of the configuration space where the two ellipses are tangent. Within this locus, we can distinguish the following points:

- Nodes. They correspond to double contacts, that is, to configurations in which both ellipses intersect in two points with multiplicity two [see Fig. 5(f)].

- Cusps. They correspond to osculating contacts, that is, to configurations in which both ellipses intersect in a point with multiplicity three [see Fig. 5(g)].

- Higher-order singularities. They correspond to hyperosculating contacts, that is, to configurations in which both ellipses intersect in a point with multiplicity four [see Fig. 5(e)]. 
Since a hyperosculating contact can be seen as the conjunction of a double and an osculating contact, a higher-order singularity can be seen as the coincidence of a cusp and a node. In the next sections, the necessary and sufficient conditions for the effective computation of cusps and nodes are presented based on their interpretation as contacts between ellipses.

Finally, it is worth noting that $\mathcal{A}$ and $\mathcal{B}$ can coincide under certain circumstances. For example, it is possible to design a $3 \mathrm{R}$ robot in which the first and the third joint axes can be aligned for some angles of the second joint angle. In this configuration the robot has a self-motion. In this case, both ellipses coincide because there are infinite solutions to the inverse kinematics.

\section{Cusps as osculating contacts}

If three of the points of intersection between $\mathcal{A}$ and $\mathcal{B}$ coincide, the ellipses are said to osculate each other at this point. In this case, the characteristic polynomial of the pencil they define is a perfect cube [23]. As a consequence,

$$
\frac{l_{3}}{l_{2}}=\frac{l_{2}}{l_{1}}=\frac{l_{1}}{l_{0}}=-\frac{1}{r}
$$

where $r$ is the triple root of the characteristic polynomial. Then, observe that

$$
\frac{l_{3}}{l_{2}}=\frac{l_{2}}{l_{1}} \Rightarrow \delta_{1}=0, \quad \frac{l_{3}}{l_{2}}=\frac{l_{1}}{l_{0}} \Rightarrow \delta_{2}=0, \quad \text { and } \quad \frac{l_{2}}{l_{1}}=\frac{l_{1}}{l_{0}} \Rightarrow \delta_{3}=0 .
$$

In other words, in a cusp not only $\Delta=0$ but all three components of the discriminant vanish. Moreover, it can be checked that

$$
l_{0} \delta_{1}=l_{1} \delta_{2}-l_{2} \delta_{3}
$$

Then, since $l_{0}=\operatorname{det}(\mathbf{B}) \neq 0(\mathcal{B}$ is a real ellipse $)$, we conclude that we have a cusp if, and only if, $\delta_{2}=0$ and $\delta_{3}=0$.

\section{Nodes as double contacts}

In 1848, Salmon gave the conditions for a double contact between two ellipses in terms of their coefficients that required the introduction of an auxiliary conic [23]. Due to Salmon's influential work, this conic is today known as the Salmon conic [27, p. 107]. Unfortunately, the application of Salmon's conditions, although expressible in terms of determinants, breaks the hierarchy of determinants of our formulation. Other methods have been proposed to avoid the use of Salmon's conic (see, for example, [28]), but probably the most convenient set of conditions for our purposes were found in 1850 by Sylvester [29]. He showed that, in a double contact, all minors of the determinant in (11) has a common root for $\lambda$. That is, in a double contact,

$$
\begin{aligned}
& \operatorname{det}\left[\lambda\left(\begin{array}{ll}
a_{1} & c_{1} \\
c_{1} & b_{1}
\end{array}\right)+\left(\begin{array}{ll}
a_{2} & c_{2} \\
c_{2} & b_{2}
\end{array}\right)\right]=p_{1} \lambda^{2}+q_{1} \lambda+r_{1}=0, \\
& \operatorname{det}\left[\lambda\left(\begin{array}{ll}
c_{1} & b_{1} \\
d_{1} & e_{1}
\end{array}\right)+\left(\begin{array}{ll}
c_{2} & b_{2} \\
d_{2} & e_{2}
\end{array}\right)\right]=p_{2} \lambda^{2}+q_{2} \lambda+r_{2}=0, \\
& \operatorname{det}\left[\lambda\left(\begin{array}{ll}
b_{1} & e_{1} \\
e_{1} & f_{1}
\end{array}\right)+\left(\begin{array}{ll}
b_{2} & e_{2} \\
e_{2} & f_{2}
\end{array}\right)\right]=p_{3} \lambda^{2}+q_{3} \lambda+r_{3}=0,
\end{aligned}
$$

where

$$
\begin{aligned}
& p_{1}=\left|\begin{array}{ll}
a_{1} & c_{1} \\
c_{1} & b_{1}
\end{array}\right|, \quad q_{1}=\left|\begin{array}{ll}
a_{1} & c_{2} \\
c_{1} & b_{2}
\end{array}\right|+\left|\begin{array}{ll}
a_{2} & c_{1} \\
c_{2} & b_{1}
\end{array}\right|, \quad r_{1}=\left|\begin{array}{ll}
a_{2} & c_{2} \\
c_{2} & b_{2}
\end{array}\right|, \\
& p_{2}=\left|\begin{array}{ll}
c_{1} & b_{1} \\
d_{1} & e_{1}
\end{array}\right|, \quad q_{2}=\left|\begin{array}{ll}
c_{1} & b_{2} \\
d_{1} & e_{2}
\end{array}\right|+\left|\begin{array}{cc}
c_{2} & b_{1} \\
d_{2} & e_{1}
\end{array}\right|, \quad r_{2}=\left|\begin{array}{ll}
c_{2} & b_{2} \\
d_{2} & e_{2}
\end{array}\right|, \\
& p_{3}=\left|\begin{array}{ll}
b_{1} & e_{1} \\
e_{1} & f_{1}
\end{array}\right|, \quad q_{3}=\left|\begin{array}{ll}
b_{2} & e_{1} \\
e_{2} & f_{1}
\end{array}\right|+\left|\begin{array}{ll}
b_{1} & e_{2} \\
e_{1} & f_{2}
\end{array}\right|, \quad r_{3}=\left|\begin{array}{ll}
b_{2} & e_{2} \\
e_{2} & f_{2}
\end{array}\right| .
\end{aligned}
$$

JMR-14-1146-Thomas-8 
Since $d_{1}$ and $e_{1}$ depend linearly on $s_{1,7}$ and $s_{2,7}$, and $f_{1}$, quadratically, equation (20) determines two possible values for $\lambda$ because $p_{1}, q_{1}$ and $r_{1}$ are constant. When substituting any of these two values in (21) and (22), the equation of a line and a conic is respectively obtained. The intersection of this line and this conic leads to up two solutions. Then, since we have two possible values for $\lambda$, we can have up to 4 nodes. Observe that closed-form explicit formulas can be derived for the computation of nodes despite the singularity locus is an octic curve.

A word of caution must be added here. Equations (20)-(22) characterize all double contacts between $\mathcal{A}$ and $\mathcal{B}$ both in the real and complex domains. As a consequence, the computed points are the nodes of the robot's singularity locus and the points where $\mathcal{A}$ and $\mathcal{B}$ have a double contact in the complex domain which we already detected as isolated roots of $\Delta=0$.

\section{Example: A 3R robot with a near hyperosculating contact}

\begin{tabular}{|c|c|c|c|c|}
\hline$i$ & $\theta_{i}$ & $d_{i}$ & $a_{i}$ & $\alpha_{i}$ \\
\hline 1 & $\theta_{1}$ & 1 & 1 & $\pi / 4$ \\
2 & $\theta_{2}$ & $1 / 2$ & $4 / 5$ & $\pi / 2$ \\
3 & $\theta_{3}$ & $5 / 3$ & 1 & 0 \\
\hline
\end{tabular}

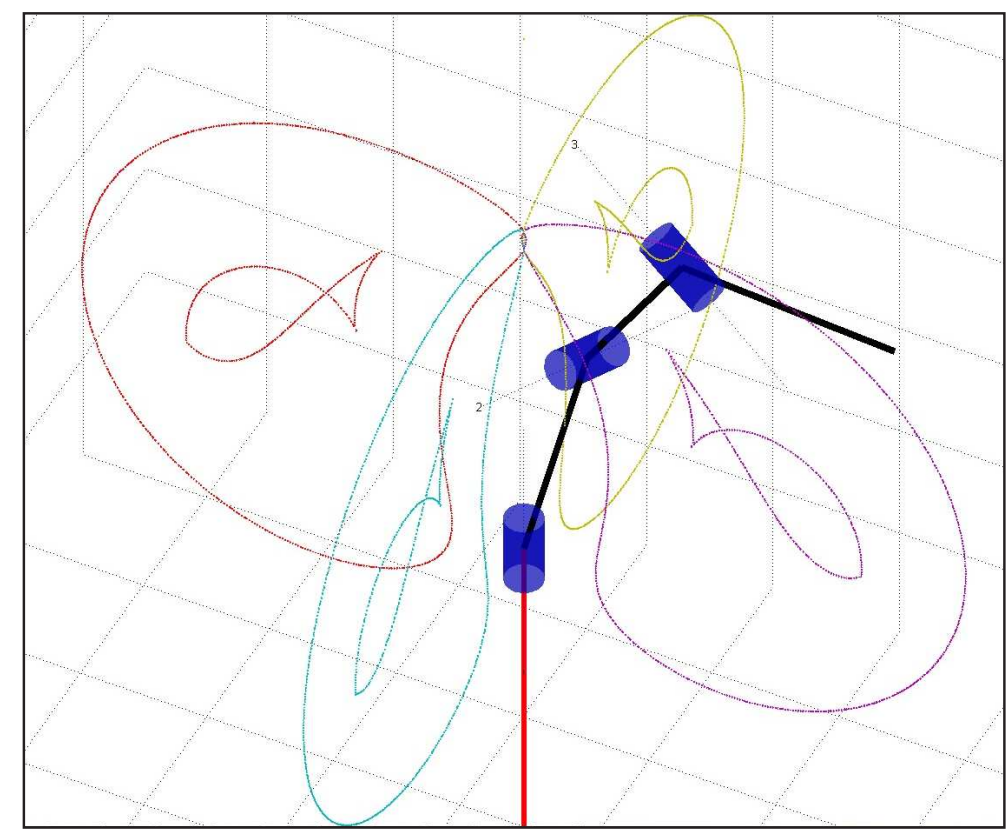

Fig. 4. The DH-parameters of the robot used as an example, and its schematic representation including orthogonal sections of its singularity locus in the robot's workspace.

Let us considered the $3 \mathrm{R}$ robot with the standard $\mathrm{DH}$ parameters shown in Fig. 4. We have taken two points on each of the three revolute axes and a point at the center of its end-effector. More specifically, $P_{1}$ and $P_{7}$ are located at the origin of the reference frame and at the center of the end-effector, respectively; $P_{2}$ and $P_{3}$ are on the common normal between the first and the second axis; $P_{4}$ and $P_{5}$ are on the common normal between the second and the third revolute axis; and $P_{6}$ is the nearest point to $P_{7}$ on the third revolute axis. For $\theta_{1}=\theta_{2}=\theta_{3}=0$ the coordinates of these points are:

$$
\begin{aligned}
& \mathbf{p}_{1}^{0}=\left(\begin{array}{lll}
0 & 0 & 0
\end{array}\right)^{T}, \\
& \mathbf{p}_{2}^{0}=\left(\begin{array}{lll}
0 & 0 & 1.6667
\end{array}\right)^{T}, \\
& \mathbf{p}_{3}^{0}=\left(\begin{array}{lll}
1 & 0 & 1
\end{array}\right)^{T}, \\
& \mathbf{p}_{4}^{0}=\left(\begin{array}{lll}
1 & -1.1785 & 2.1785
\end{array}\right)^{T}, \\
& \mathbf{p}_{5}^{0}=\left(\begin{array}{lll}
1.8 & -0.3536 & 1.3536
\end{array}\right)^{T}, \\
& \mathbf{p}_{6}^{0}=\left(\begin{array}{lll}
1.8 & -1.5321 & 0.1750
\end{array}\right)^{T}, \\
& \mathbf{p}_{7}^{0}=\left(\begin{array}{lll}
2.8 & -1.5321 & 0.1750
\end{array}\right)^{T}
\end{aligned}
$$




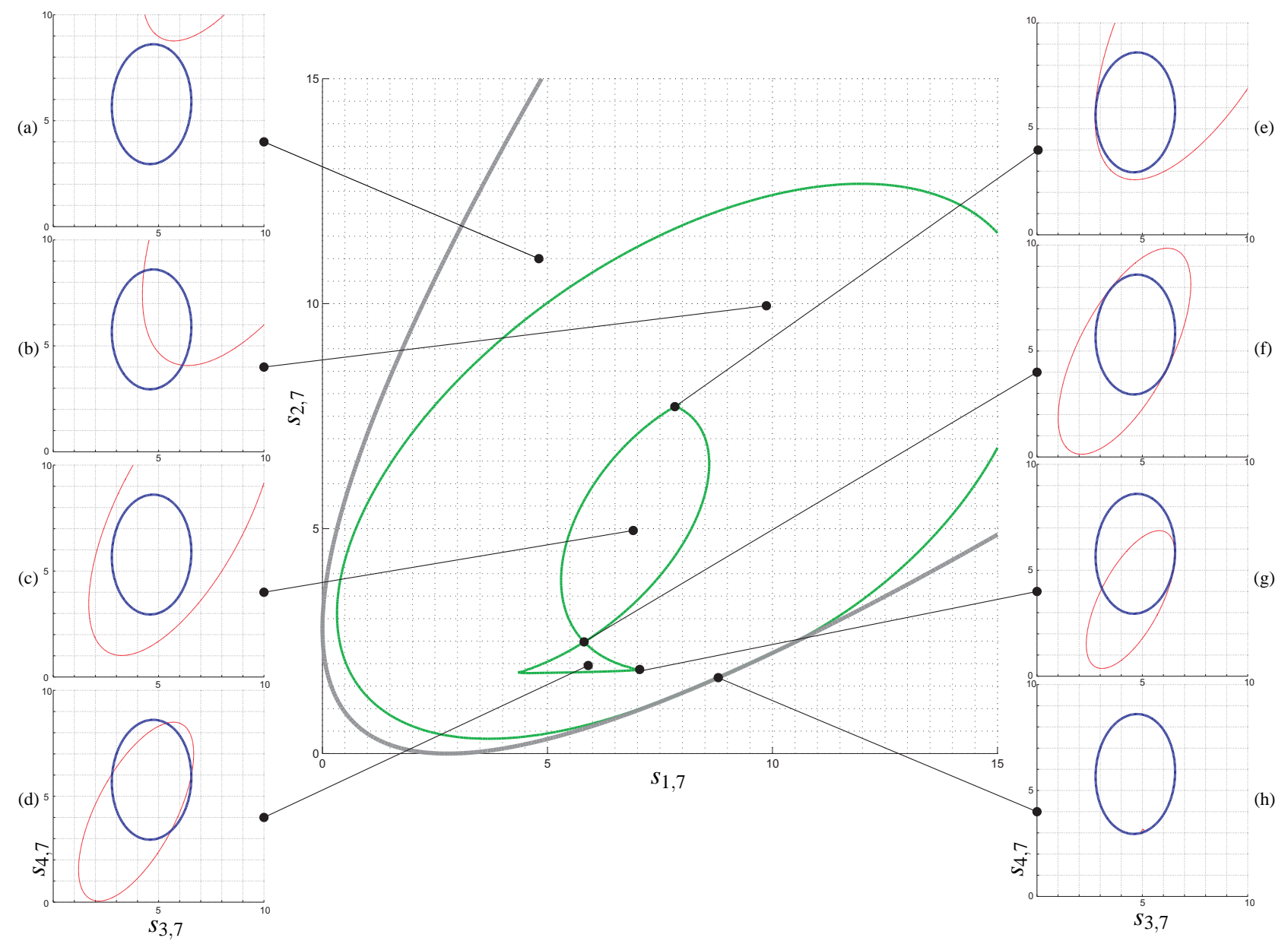

Fig. 5. Center: Plot of the curves defined by $\operatorname{det}(\mathbf{A})=0$ (in gray) and $\Delta=0$ (in green). These curves segment the plane into regions where the spatial relationship between $\mathcal{A}$ and $\mathcal{B}$ is the same. We are only interested in the region where $\mathcal{A}$ is a real ellipse (that is, the region where $\operatorname{det}(\mathbf{A}) \leq 0$ ). Left column: spatial relationships between $\mathcal{A}$ (in red) and $\mathcal{B}$ (in blue) associated with different regions of this plane. Right column: spatial relationships between $\mathcal{A}$ and $\mathcal{B}$ in different points of the singularity locus.

Substituting the squared distances between the above points appearing in the expressions for $\mathcal{A}$ and $\mathcal{B}$, we obtain:

$$
\begin{aligned}
& \mathcal{A}:\left(\begin{array}{lll}
s_{3,7} & s_{4,7} & 1
\end{array}\right)\left(\begin{array}{ccc}
26.54 & -11.11 & d_{1} \\
-11.11 & 11.11 & e_{1} \\
d_{1} & e_{1} & f_{1}
\end{array}\right)\left(\begin{array}{c}
s_{3,7} \\
s_{4,7} \\
1
\end{array}\right)=0 \\
& \mathcal{B}:\left(\begin{array}{lll}
s_{3,7} & s_{4,7} & 1
\end{array}\right)\left(\begin{array}{ccc}
22.23 & -0.63 & -100.15 \\
-0.63 & 9.89 & -54.2 \\
-100.15 & -54.2 & 701.71
\end{array}\right)\left(\begin{array}{c}
s_{3,7} \\
s_{4,7} \\
1
\end{array}\right)=0
\end{aligned}
$$

where $d_{1}, e_{1}$, and $f_{1}$ depend on $s_{1,7}$ and $s_{2,7}$ as follows:

$$
\begin{aligned}
\left(\begin{array}{l}
d_{1} \\
e_{1}
\end{array}\right) & =\left(\begin{array}{ccc}
-14.03 & -1.4 & 30.085 \\
7.86 & -7.86 & -35.23
\end{array}\right)\left(\begin{array}{c}
s_{1,7} \\
s_{2,7} \\
1
\end{array}\right) \\
f_{1} & =\left(\begin{array}{lll}
s_{1,7} & s_{2,7} & 1
\end{array}\right)\left(\begin{array}{ccc}
13.58 & -7.407 & -38.28 \\
-7.407 & 16.667 & 12.56 \\
-38.28 & 12.56 & 156.28
\end{array}\right)\left(\begin{array}{c}
s_{1,7} \\
s_{2,7} \\
1
\end{array}\right)
\end{aligned}
$$




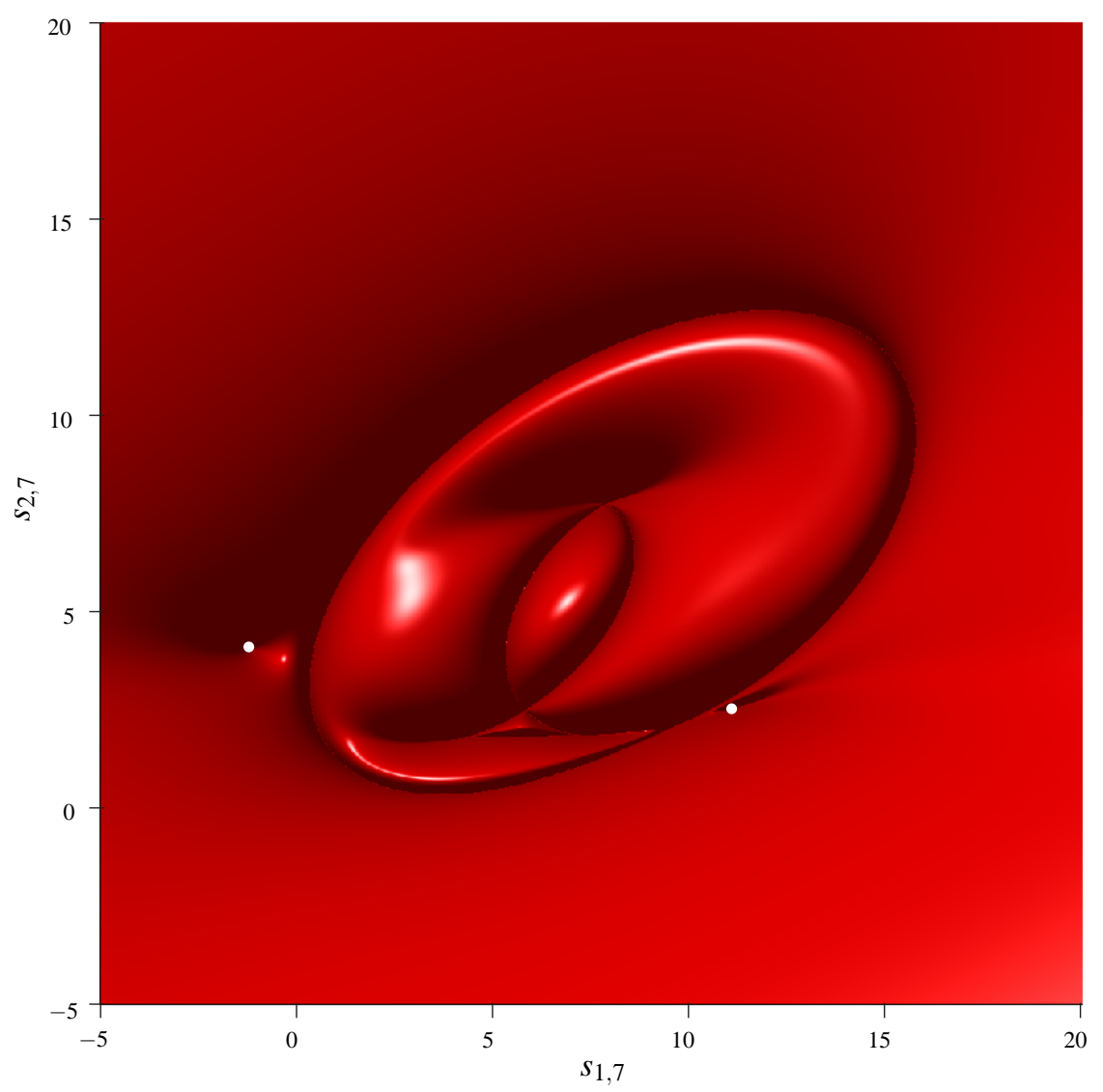

Fig. 6. Shaded depth map of $\log \left(\operatorname{abs}\left(\Delta\left(s_{1,7}, s_{2,7}\right)\right)\right)$. The robot's singularity locus appear as valleys of this map. The two points marked with white dots correspond to configurations unreachable by the robot where $\mathcal{A}$ and $\mathcal{B}$ have a double contact in the complex domain.

Now, substituting in (12)-(15), we obtain:

$$
\begin{aligned}
& l_{3}=952.6 s_{1,7}^{2}-1905.2 s_{1,7} s_{2,7}-5292.2 s_{1,7}+952.6 s_{2,7}^{2}-5292.2 s_{2,7}+7350.3 \\
& l_{2}=1183.2 s_{1,7}^{2}-1703.5 s_{1,7} s_{2,7}-8745.4 s_{1,7}+2284.4 s_{2,7}^{2}-14564.0 s_{2,7}+28656.0 \\
& l_{1}=993.53 s_{1,7}^{2}-1083.8 s_{1,7} s_{2,7}-8540.5 s_{1,7}+1219.3 s_{2,7}^{2}-5762.8 s_{2,7}+14744.0 \\
& l_{0}=-17342.0
\end{aligned}
$$

Then, substituting these values in (17), we finally obtain:

$$
\begin{aligned}
\delta_{1}= & -4.53422 \cdot 10^{5} s_{1,7}^{4}+1.1056 \cdot 10^{6} s_{1,7}^{3} s_{2,7}+7.3007 \cdot 10^{6} s_{1,7}^{3}-4.1345 \cdot 10^{6} s_{1,7}^{2} s_{2,7}^{2}+1.5926 \cdot 10^{7} s_{1,7}^{2} s_{2,7}-7.7745 \cdot 10^{7} s_{1,7}^{2} \\
& +4.4273 \cdot 10^{6} s_{1,7} s_{2,7}^{3}-7.535 \cdot 10^{6} s_{1,7} s_{2,7}^{2}-1.1746 \cdot 10^{8} s_{1,7} s_{2,7}+3.6041 \cdot 10^{8} s_{1,7}-4.057 \cdot 10^{6} s_{2,7}^{4}+5.4596 \cdot 10^{7} s_{2,7}^{3} \\
& -2.8952 \cdot 10^{8} s_{2,7}^{2}+7.1428 \cdot 10^{8} s_{2,7}-7.1279 \cdot 10^{8}, \\
\delta_{2}= & -1.1755 \cdot 10^{6} s_{1,7}^{4}+2.9748 \cdot 10^{6} s_{1,7}^{3} s_{2,7}+1.8793 \cdot 10^{7} s_{1,7}^{3}-5.5586 \cdot 10^{6} s_{1,7}^{2} s_{2,7}^{2}-2.7397 \cdot 10^{6} s_{1,7}^{2} s_{2,7}-1.3712 \cdot 10^{8} s_{1,7}^{2} \\
& +4.553 \cdot 10^{6} s_{1,7} s_{2,7}^{3}+4.572 \cdot 10^{6} s_{1,7} s_{2,7}^{2}-8.5565 \cdot 10^{7} s_{1,7} s_{2,7}+4.6545 \cdot 10^{8} s_{1,7}-2.7854 \cdot 10^{6} s_{2,7}^{4}+3.0922 \cdot 10^{7} s_{2,7}^{3} \\
& -1.6907 \cdot 10^{8} s_{2,7}^{2}+4.7164 \cdot 10^{8} s_{2,7}-5.4996 \cdot 10^{8}, \\
\delta_{3}= & -987099.0 s_{1,7}^{4}+2.1537 \cdot 10^{6} s_{1,7}^{3} s_{2,7}+1.697 \cdot 10^{7} s_{1,7}^{3}-3.5976 \cdot 10^{6} s_{1,7}^{2} s_{2,7}^{2}-7.0623 \cdot 10^{6} s_{1,7}^{2} s_{2,7}-1.2275 \cdot 10^{8} s_{1,7}^{2} \\
& +2.6431 \cdot 10^{6} s_{1,7} s_{2,7}^{3}+8.3354 \cdot 10^{6} s_{1,7} s_{2,7}^{2}-3.6933 \cdot 10^{7} s_{1,7} s_{2,7}+4.035 \cdot 10^{8} s_{1,7}-1.4868 \cdot 10^{6} s_{2,7}^{4}+1.4053 \cdot 10^{7} s_{2,7}^{3} \\
& -1.0878 \cdot 10^{8} s_{2,7}^{2}+4.2249 \cdot 10^{8} s_{2,7}-7.1432 \cdot 10^{8} .
\end{aligned}
$$

The robot's singularity locus, according to (16) is given by $\Delta=4 \delta_{1} \delta_{3}-\delta_{2}^{2}=0$. In Fig. 5, we can see the curves defined by $\Delta=0$ and $l_{3}=\operatorname{det}(\mathbf{A})=0$. The region determined by $l_{3}<0$ is the region where $\mathcal{A}$ is a real ellipse. Within this region, 
the curve determined by $\Delta=0$ segments the plane into regions with the same number of inverse kinematics solutions. For example, in Fig. 5, we can identify the following regions:

(a) A region with no solution to the inverse kinematics. Both ellipses are disjoint.

(b) A region with two solutions to the inverse kinematics. Both ellipses intersect at two real points.

(c) A region with no solution to the inverse kinematics. One ellipse contains the other. This region corresponds to a void in the workspace.

(d) A region with four solutions to the inverse kinematics. Both ellipses intersect at four real points.

At this point we must remember that $\Delta=0$ also contains the points where $\mathcal{A}$ and $\mathcal{B}$ have a double contact in the complex domain. To visualize these isolated points unreachable by the robot, it is better to represent $\Delta$ as a two dimensional function (see Fig. 6).

Within the singularity locus (points where both ellipses are tangent in the real domain) we can identify in Fig. 5 the following interesting points:

(e) A higher-order singularity. A point where both ellipses have a hyperosculating contact.

(f) A node. A point where both ellipses have a double point contact.

(g) A cusp. A point where both ellipses have an osculating contact.

(h) A point where the singularity locus is tangent to the boundary of the region where $\mathcal{A}$ is a real ellipse. At this singularity point $\mathcal{A}$ degenerates in a point because $\operatorname{det}(A)=0$. As proved in Section II, this corresponds to a singularity in which the robot's end-effector is aligned with the first rotation axis.

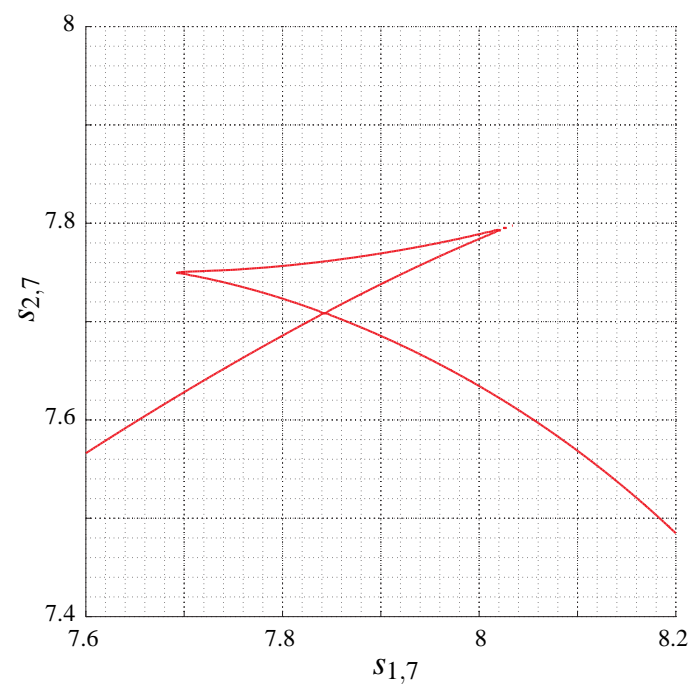

Fig. 7. What it seemed to be a higher-order singularity in Fig. 5, it is revealed to be a node close to meet two cusps.

In general, to correctly observe cusps, nodes or tangencies, we need a very particular choice of ranges of variables and parameters and a suitable aspect ratio of the graphics. This is a typical problem when using computer graphics for the study of singularities [30]. For example, if we magnify the region where the singularity locus seems to have a higher-order singularity, we observe that we actually have is a node close to two cusps (Fig. 7). Likewise, if we magnify the region where the singularity locus is tangent to the curve defined by $l_{3}=\operatorname{det}(\mathbf{A})=0$, we actually observe two tangencies very close to each other. This is better analyzed by mapping the singularity locus onto the robot's workspace as explained next.

We have obtained the singularity locus in the plane $\left(s_{1,7}, s_{2,7}\right)$, but mapping this locus onto the robot's workspace is straightforward by observing that

$$
z=\frac{s_{1,2}-s_{2,7}+s_{1,7}}{2 \sqrt{s_{1,2}}}, \quad \rho=+\sqrt{s_{2,7}-\left(d_{1,2}-z\right)^{2}},
$$

whose inverse mapping is given by:

$$
s_{1,7}=\rho^{2}+z^{2}, \quad s_{2,7}=\left(d_{1,2}-z\right)^{2}+\rho^{2} .
$$




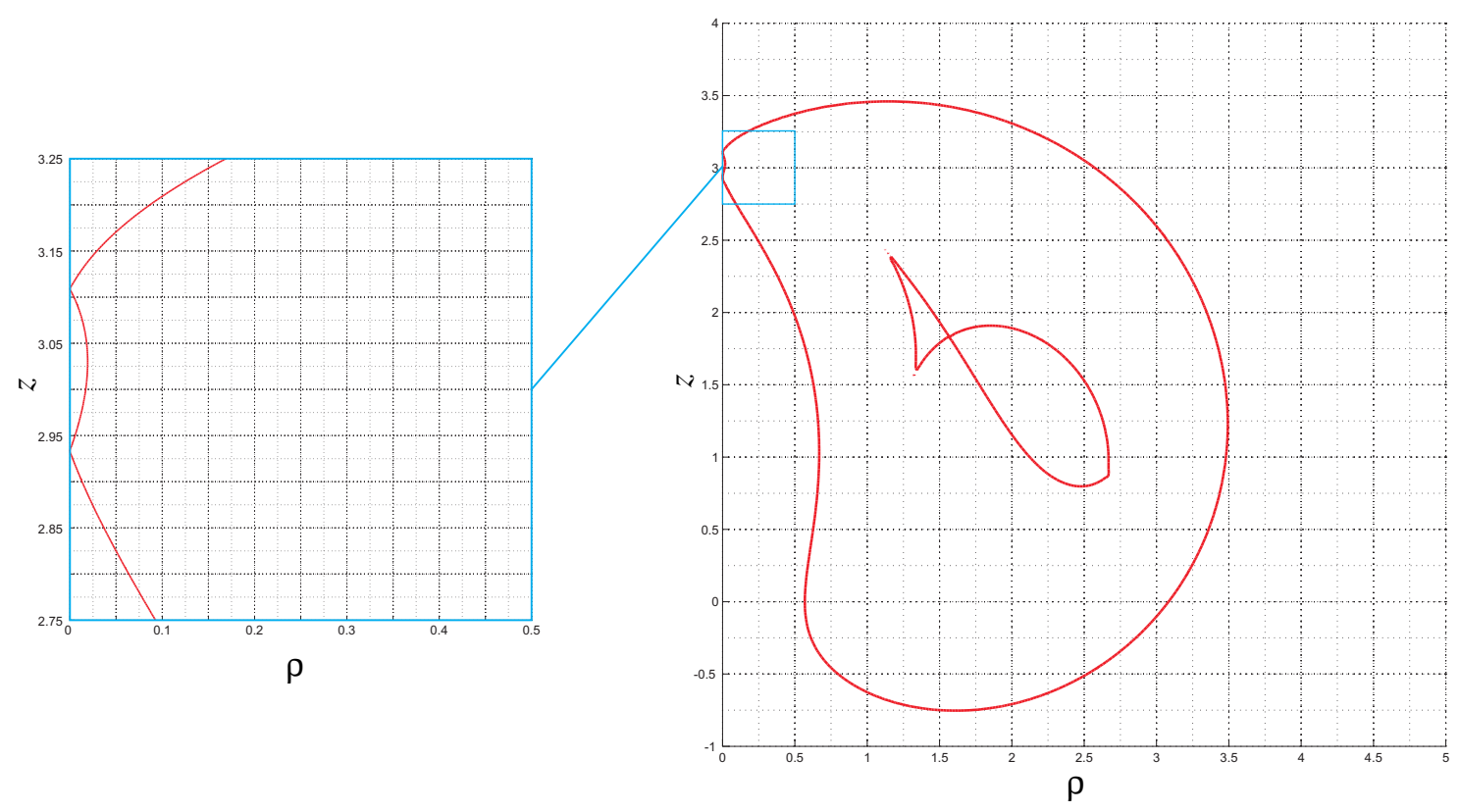

Fig. 8. The singularity locus shown in Fig. 5(center) mapped onto the robot's workspace $(\rho, z)$. The two singularities at $\rho=0$ correspond to the two tangencies between the curves defined by $\Delta=0$ and $\operatorname{det}(\mathbf{A})=0$ in the distance space $\left(s_{1,7}, s_{2,7}\right)$.

The result is presented in Fig. 8. In this plot, we effectively observe two singular points with $\rho=0$ that correspond to the two tangencies between $\Delta=0$ and $\operatorname{det}(\mathbf{A})=0$ in the distance space $\left(s_{1,7}, s_{2,7}\right)$.

The curves defined by $\delta_{2}=0$ and $\delta_{3}=0$ are plotted in Fig. 9. Observe how their intersection coincide with the cusps of the singularity locus plotted in Fig. 5, as expected. The system of equations $\delta_{2}=\delta_{3}=0$ has 12 solutions, 8 of them are complex. The real solutions correspond to the coordinates in the plane $\left(s_{1,7}, s_{2,7}\right)$ of the 4 cusps: $(7.3578,1.8467)$, (7.6732,7.7518), $(4.0859,1.7505)$, and $(8.044,7.8009)$. The coordinates of these points in the robot's workspace through the mapping given in (30) are $(1.0837,2.4867),(2.6491,0.8098),(1.3164,1.5340)$, and $(2.6875,0.9063)$, respectively.

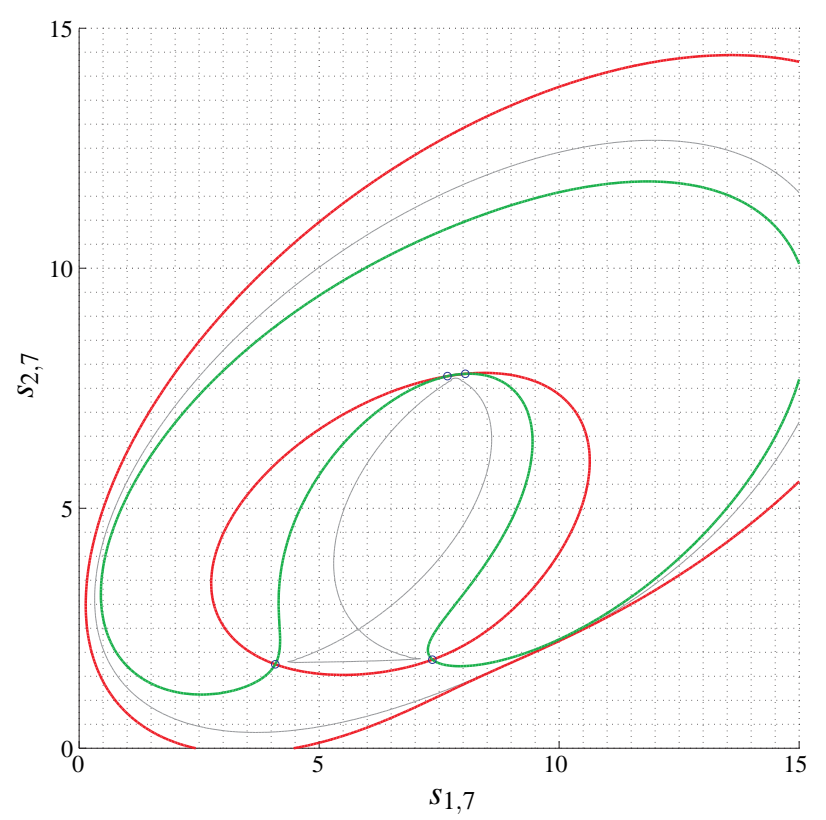

Fig. 9. Plot of $\delta_{2}=0$ (in red) and $\delta_{3}=0$ (in green). Observe how both curves intersect at the cusps of the singularity locus (light gray). 

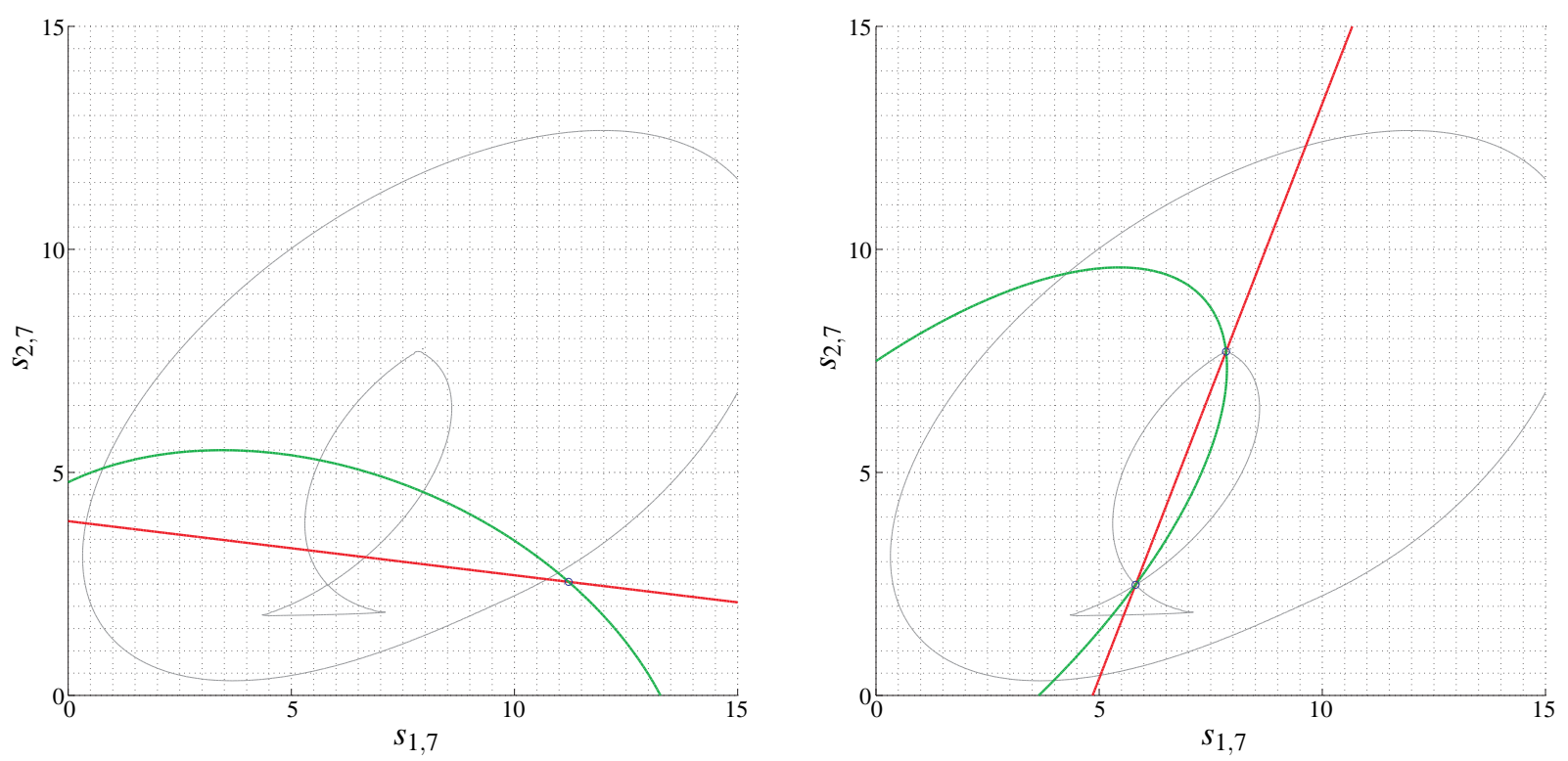

Fig. 10. Left: Plot of the line (in red) and ellipse (in green) resulting from substituting $\lambda_{1}=-2.3439$ in (33) and (34), respectively. Right: The same for $\lambda_{2}=-0.5461$. Observe in the first case the intersection points do not lie in the singularity locus represented in light gray.

Concerning the computation of the nodes, the evaluation of (20)-(22) yields:

$$
\begin{array}{r}
171.4678 \lambda^{2}+495.5418 \lambda+219.4787=0 \\
\left(68.587 s_{1,7}+102.88 s_{2,7}+57.156\right) \lambda^{2}+\left(133.79 s_{1,7}+18.816 s_{2,7}+1439.7\right) \lambda+1024.5=0 \\
\left(89.163 s_{1,7}^{2}-41.152 s_{1,7} s_{27}-297.21 s_{1,7}+123.46 s_{2,7}^{2}-274.35 s_{2,7}+495.35\right) \lambda^{2}+ \\
\left(134.29 s_{1,7}^{2}-146.5 s_{1,7} s_{2,7}+94.598 s_{1,7}+164.81 s_{2,7}^{2}-603.29 s_{2,7}+5522.9\right) \lambda+4000.7=0
\end{array}
$$

The roots of (32) are $\lambda_{1}=-2.3439$ and $\lambda_{2}=-0.5461$. The substitution of each of these two values in (33) and (34) leads to the equation of a line and a conic, respectively (see Fig. 10). For the first root, the coordinates of the two intersection points of the line and the conic are $(11.21,2.55)$ and $(-1.15,4.05)$. Observe how they correspond to the points, shown in Fig. 6, where $\mathcal{A}$ and $\mathcal{B}$ have a double contact in the complex domain. For the second root, the coordinates are $(7.84,7.71)$ and $(5.81,2.48)$. In this case, they correspond to double contacts in the real domain, that is, they are effectively nodes of the robot's singularity locus. When mapped onto the robot's workspace, their coordinates are $(2.6606,0.8723)$ and $(1.5661,1.8323)$, respectively.

\section{Conclusions}

It has been shown how the singularity loci of $3 \mathrm{R}$ robots can be described in terms of a hierarchy of four levels of determinants where the entries of the determinants at the lowest level are squared distances between points in the robot's workspace. This coordinate-free formulation, where no reference frames are required, has lead to two new results concerning the cusps and the nodes in the singularity loci of $3 \mathrm{R}$ robots:

- Cusps can be obtained as the intersection of two quartic curves, a simpler characterization than the traditional one based on imposing three equal roots to the inverse kinematics polynomial.

- Nodes are constructible. That is, they can be obtained by arithmetic operations and taking square roots a finite number of times.

Higher-order singularities can be seen as those singularities in which a node coincides with a cusp. These higher-order singularities have already been classified into lips, beaks and swallowtail singularities [31]. Actually, the near hyperosculating contact analyzed in the example corresponds to a swallowtail singularity. The identification of these three kinds of higher-order singularities using the presented Distance Geometry formulation is a point that deserves further attention.

\section{References}

[1] P. Wenger, "Cuspidal and non-cuspidal robot manipulators,” Robotica, Vol. 25, No. 6, pp. 677-689, 2007. 
[2] P. Wenger, "Some guidelines for the kinematic design of new manipulators," Mechanism and Machine Theory, Vol. 35, No. 3, pp. 437-449, 2000.

[3] R. Paul and H. Zhang, "Computationally efficient kinematics for manipulators with spherical wrists based on the homogeneous transformation representation,” The International Journal of Robotics Research, Vol. 5, No. 2, pp. 32-44, 1986.

[4] D.L. Pieper, The Kinematics of Manipulators under Computer Control, PhD thesis, Stanford University, Department of Mechanical Engineering, 1968.

[5] P. Kovács and G. Hommel, "On the tangent-half-angle substitution,” In: Computational Kinematics, J. Angeles, G. Hommel, and P. Kovács (Eds.), Kluwer Academic Publishers, Dordrecht, pp. 27-39, 1993.

[6] D.R. Smith and H. Lipkin, "Higher order singularities of regional manipulators," Proceedings of the IEEE International Conference on Robotics and Automation, Vol. 1, pp. 194-199, 1993.

[7] D.R. Smith and H. Lipkin, "Kinematic analysis of solvable manipulators using conic sections," Proc. 21st ASME Mechanisms Conference, pp. 16-19, 1990.

[8] M. Ceccarelli, "On the workspace of 3R robot arms," Proc. 5th IFToMM International Symposium on Theory and Practice of Mechanism, Bucharest, Vol. II-1, pp. 37-46, 1989.

[9] M. Husty, E. Ottaviano and M. Ceccarelli, "A geometrical characterization of workspace singularities in 3R manipulators," in Advances in Robot Kinematics: Analysis and Design, J. Lenarčič, and Ph. Wenger (eds.), pp. 411-418, Springer, 2008.

[10] M. Zein, Analyse cinématique des manipulateurs sériels $3 R$ orthogonaux et des manipulateurs parallèles plans, PhD dissertation, École Central de Nantes, Université de Nantes, 2007.

[11] H. Bamberger, A. Wolf, M. Shoham, "Assembly Mode Changing in Parallel Mechanisms," IEEE Trans. on Robotics, Vol. 24, No. 4, pp. 765-772, 2008.

[12] S.F.P. Saramago, E. Ottaviano, and M. Ceccarelli, "A characterization of the workspace boundary of three-revolute manipulators," Proc. of DECT'02, ASME 2002 Design Engineering Technical Conferences, Montreal, Canada, 2002.

[13] P. Wenger, "Design of cuspidal and non-cuspidal robot manipulators," Proceedings of the 1997 IEEE International Conference on Robotics and Automation, pp. 2172-2177, 1997.

[14] M. Baili, Analyse et classification de manipulateurs $3 R$ à axes orthogonaux, PhD dissertation, École Central de Nantes, Université de Nantes, 2004.

[15] P. Donelan and A. Müller, "General formulation of the singularity locus for a 3-dof regional manipulator," Proc. IEEE International Conference on Robotics and Automation, 2011.

[16] F. Thomas, "Computing cusps of 3R robots using Distance Geometry," 14th International Symposium on Advances in Robot Kinematics (ARK2014), Ljubljana, Slovenia, Jun 29-Jul 3, 2014.

[17] F. Thomas and L. Ros, "Revisiting trilateration for robot localization," IEEE Transactions on Robotics, Vol. 21, No. 1, pp. 93-101, 2005.

[18] W.M. Faucette, "A geometric interpretation of the solution of the general quartic polynomial," The American Mathematical Monthly, Vol. 103, No. 1, pp. 51-57, 1996.

[19] M. Bôcher, Plane Analytic Geometry, Henry Holt and Co., 1915.

[20] Y-K. Choi, W. Wang, Y. Liu, and M-S. Kim, "Continuous collision detection for two moving elliptic disks," IEEE Transactions on Robotics, Vol. 22, No. 2, pp. 213-224, 2006.

[21] D.M.Y. Sommerville, Analytical Conics, G. Bell \& Sons, 1961.

[22] J. Richter-Gebert, Perspectives on Projective Geometry: A Guided Tour Through Real and Complex Geometry, Springer, 2011.

[23] G. Salmon, A Treatise on Conic Sections, Chelsea Publishing Co., 1869.

[24] B. Elizalde, M. Alberich-Carramiñana, and F. Thomas, "On the relative position of two coplanar ellipses," in preparation.

[25] L.E. Dickson, Elementary Theory of Equations, Wiley, New York, 1914.

[26] J.F. Blinn, "Polynomial discriminants I. Matrix magic," IEEE Computer Graphics and Applications, Vol. 20, No. 6, pp. 94-98, 2002.

[27] I.V. Dolgachev, Classical Algebraic Geometry: A Modern View, Cambridge University Press, 2012.

[28] C.N. Srinivasiengar, "On the conditions for the double contact of two conics," Journal of the Mysore University, Vol. 1, No. 2, pp. 110-111, 1927.

[29] J.J. Sylvester, “Additions to the Articles 'On a New Class of Theorems' and 'On Pascal's Theorem," Philosophical Magazine, pp. 363-370, 1850. Reprinted in J. J. Sylvester's Mathematical Papers, Vol. 1, Cambridge, University Press, pp. 145-151, 1904.

[30] R. Morris, "The use of computer graphics for solving problems in singularity theory," Visualization and Mathematics, Experiments, Simulations and Environments, H. C. Hege and K. Polthier (eds.), pp. 53-66.Springer, 1997.

[31] F. Thomas and P. Wenger, "On the topological characterization of robot singularity loci. A catastrophe-theoretic approach,” Proc. IEEE International Conference on Robotics and Automation, pp. 3940-3945, 2011. 


\section{Figure captions}

- Figure 1. A 3R robot and associated notation.

- Figure 2. Encircling a cusp (left) or an $\alpha$-curve (right) allows, under some circumstances, to change the working mode of a robot without trespassing a singularity. The curves in red represent part of the singularity loci.

- Figure 3. Left: The equivalent bar-and-joint framework associated with the 3R robot in Fig. 1. Right: This framework can be split by the plane defined by $P_{3}, P_{4}$ and $P_{7}$ into two subassemblies, each containing a tetrahedron (shown as a shaded volume) and a triangle.

- Figure 4. The DH-parameters of the robot used as an example, and its schematic representation including orthogonal sections of its singularity locus in the robot's workspace.

- Figure 5. Center: Plot of the curves defined by $\operatorname{det}(\mathbf{A})=0$ (in gray) and $\Delta=0$ (in green). These curves segment the plane into regions where the spatial relationship between $\mathcal{A}$ and $\mathcal{B}$ is the same. We are only interested in the region where $\mathcal{A}$ is a real ellipse (that is, the region where $\operatorname{det}(\mathbf{A}) \leq 0$ ). Left column: spatial relationships between $\mathcal{A}$ (in red) and $\mathcal{B}$ (in blue) associated with different regions of this plane. Right column: spatial relationships between $\mathcal{A}$ and $\mathcal{B}$ in different points of the singularity locus.

- Figure 6. Shaded depth map of $\log \left(\operatorname{abs}\left(\Delta\left(s_{1,7}, s_{2,7}\right)\right)\right)$. The robot's singularity locus appear as valleys of this map. The two points marked with white dots correspond to configurations unreachable by the robot where $\mathcal{A}$ and $\mathcal{B}$ have a double contact in the complex domain.

- Figure 7. What it seemed to be a higher-order singularity in Fig. 5, it is revealed to be a node close to meet two cusps.

- Figure 8. The singularity locus shown in Fig. 5(center) mapped onto the robot's workspace $(\rho, z)$. The two singularities at $\rho=0$ correspond to the two tangencies between the curves defined by $\Delta=0$ and $\operatorname{det}(\mathbf{A})=0$ in the distance space $\left(s_{1,7}, s_{2,7}\right)$

- Figure 9. Plot of $\delta_{2}=0$ (in red) and $\delta_{3}=0$ (in green). Observe how both curves intersect at the cusps of the singularity locus (light gray).

- Figure 10. Left: Plot of the line (in red) and ellipse (in green) resulting from substituting $\lambda_{1}=-2.3439$ in (33) and (34), respectively. Right: The same for $\lambda_{2}=-0.5461$. Observe in the first case the intersection points do not lie in the singularity locus represented in light gray. 